PROCEEDINGS OF THE

AMERICAN MATHEMATICAL SOCIETY

Volume 86, Number 1, September 1982

\title{
THE GLOBAL DIMENSION OF FBN RINGS WITH ENOUGH CLANS
}

\author{
ROBERT F. DAMIANO
}

\begin{abstract}
For an FBN ring $R$, a classical set of prime ideals $\left\{P_{1}, \ldots, P_{n}\right\}$ is one for which the semiprime ideal $N=\bigcap_{i=1}^{n} P_{i}$ satisfies the Artin-Rees property. A minimal classical set is called a clan. We say an FBN ring $R$ has enough clans if each prime ideal $P$ is an element of a clan. In this paper, we show that for such rings the Krull dimension is less than or equal to the global dimension.
\end{abstract}

For a commutative noetherian ring $R$, it is well known that the Krull dimension of the ring is less than or equal to the global dimension of the ring [2, Lemma 3.1]. This same inequality can be conjectured to be true for all noetherian rings without the commutativity hypothesis. Some progress in this direction has been made, especially in the case of noetherian rings satisfying a polynomial identity [8, 11]. In this paper, we show that the conjecture is true for FBN rings with enough clans.

THEOREM 1. If $R$ is an FBN ring with enough clans, then $K-\operatorname{dim}(R) \leq$ $\operatorname{gl} \operatorname{dim}(R)$.

We first prove a proposition that will allow us to assume without loss of generality that $R$ is a semilocal FBN ring with a unique maximal clan. Let $R_{[P]}$ be the localization at the semiprime ideal associated to the clan containing the prime ideal $P$. We note that by $[9$, Theorem 5] a clan contains only a finite number of prime ideals. Thus $R_{[P]}$ is a semilocal noetherian ring.

PROPOSITION. If $R$ is an FBN ring with enough clans such that $[P]$ is the clan containing the prime ideal $P$, then $R_{[P]}$ is an FBN ring with enough clans. Moreover

(i) $K-\operatorname{dim}(R)=\sup \left\{K-\operatorname{dim}\left(R_{[M]}\right) \mid M\right.$ a maximal ideal $\}$.

(ii) $\operatorname{gl} \operatorname{dim}(R)=\sup \left\{\operatorname{gl} \operatorname{dim} R_{[M]}\right) \mid M$ a maximal ideal $\}$.

PROOF. The proof that $R_{[P]}$ is an FBN ring with enough clans can be found in [13, Proposition 2]. Pick a proper chain of prime ideals $P_{0} \subseteq P_{1} \subseteq \cdots \subseteq M$ where $M$ is the maximal prime ideal of the chain. Localize $R$ at the clan containing $M$, i.e. $[M]$. Thus $R_{[M]} \otimes P_{0} \subseteq R_{[M]} \otimes P_{1} \subseteq \cdots \subseteq R_{[M]} \otimes M$ is a proper chain of prime ideals in $R_{[M]}$. It follows that

$$
K-\operatorname{dim}(R)=\sup \left\{K-\operatorname{dim}\left(R_{[M]}\right) \mid M \text { a maximal ideal }\right\} .
$$

Let $N$ be an $R_{[M]}$-module with $R_{[M]}$-injective resolution

$$
0 \rightarrow N \rightarrow E_{0} \rightarrow E_{1} \rightarrow E_{2} \rightarrow \cdots
$$

Received by the editors September 22, 1981.

1980 Mathematics Subject Classification. Primary 16A33, 16A60.

(c) 1982 American Mathematical Society 0002-9939/81/0000-0791/\$02.00 
Since localization at $[M]$ is a perfect flat epimorphism [14, Proposition 2.3.5], this is also an $R$-injective resolution of $N$ viewed as an $R$-module. Hence,

$$
\sup \left\{\operatorname{gl} \operatorname{dim}\left(R_{[M]}\right)\right\} \leq \operatorname{gl} \operatorname{dim}(R) .
$$

Suppose $n=\sup \left\{\operatorname{gl} \operatorname{dim}\left(R_{[M]}\right)\right\}<\operatorname{gl} \operatorname{dim}(R)$. Pick an $R$-module $K$ such that inj $\operatorname{dim}(K)>n$. If $E_{n+1}$ is the $n+1$-injective in a minimal injective resolution of $K$, there exists an $0 \neq x \in E_{n+1}$ such that $\operatorname{Ann}_{R}(R x)=P$, a prime ideal. Let $M^{\prime} \supseteq P$ be a maximal ideal. By [4, Proposition 1], localizing the injective resolution of $K$ at $\left[M^{\prime}\right]$ produces an $R_{\left[M^{\prime}\right]}$-injective resolution of $R_{\left[M^{\prime}\right]} \otimes K$ of length greater than $n$. This contradiction gives the equality of (ii).

PROOF OF THEOREM 1. By the Proposition, it suffices to assume $R$ has been localized at a maximal clan. Let $J=\bigcap\left\{M_{i} \mid M_{i} \in[M]\right\}$ be the Jacobson radical of $R$. By [10, Corollary 3.8], $J$ satisfies the Artin-Rees property. We note that by [7, Corollary 3.8], the Krull dimension of $R$ is finite. Therefore, without loss of generality, assume the global dimension of $R$ is finite. We now prove the result by induction on the global dimension.

If $\operatorname{gl} \operatorname{dim}(R)=0$, then $R$ is semisimple and we are done. Suppose that the result is true for global dimension less than or equal to $k-1$ and let $R$ be an FBN ring with enough clans and global dimension $k$. Let $P$ be a prime ideal of $R$ such that $K-\operatorname{dim}\left(R_{[P]}\right)=K-\operatorname{dim}(R)-1$. Let $N=\bigcap\left\{P_{i} \mid P_{i} \in[P]\right\}$. Since $P_{i} \in[P]$ has the property that $K-\operatorname{dim}\left(R / P_{i}\right)=1[9$, Corollary 6$]$, we can pick $0 \neq x \in J$ such that $\bar{x}$ is regular in the ring $R / N$. Consider the exact sequence

$$
0 \rightarrow R / N \stackrel{x}{\rightarrow} R / N \rightarrow R / R x+N \rightarrow 0
$$

where the monomorphism is given by right multiplication by $x$.

This sequence gives rise to the long exact sequence

$$
\begin{aligned}
\cdots \rightarrow \operatorname{Ext}_{R}^{k-1}(R / N, R / N) & \rightarrow \operatorname{Ext}_{R}^{k-1}(R / N, R / R x+N) \\
& \rightarrow \operatorname{Ext}_{R}^{k}(R / N, R / N) \stackrel{x}{\rightarrow} \operatorname{Ext}_{R}^{k}(R / N, R / N) \\
& \rightarrow \operatorname{Ext}_{R}^{k}(R / N, R / R x+N) \rightarrow 0
\end{aligned}
$$

where the map from $\operatorname{Ext}_{R}^{k}(R / N, R / N)$ to $\operatorname{Ext}_{R}^{k}(R / N, R / N)$ is given by right multiplication by $x$.

We first claim that $\operatorname{Ext}_{R}^{k}(R / N, R / J)=0$. Suppose $\operatorname{Ext}_{R}^{k}(R / N, R / J) \neq 0$. Since $J$ has the Artin-Rees property and $R$ is a semilocal FBN ring, the minimal injective resolution of $R / J$ has the property that $E_{i} \cong \bigoplus E\left(T_{j}\right)$ where $T_{j}$ are simple $R$-modules [9, Theorem 5]. Moreover, every finitely generated submodule of $E_{i}$ has finite length [6, Proposition 4.3].

Let

$$
0 \neq \bar{\varphi} \in \operatorname{Ext}_{R}^{k}(R / N, R / J)=\operatorname{ker} d_{k}^{*} / \operatorname{Im} d_{k-1}^{*} .
$$

Pick $\varphi \in \operatorname{Hom}_{R}\left(R / N, E_{k}\right)$ such that $\varphi \in \operatorname{ker} d_{k}^{*}$ and $\varphi+\operatorname{Im} d_{k-1}^{*}=\bar{\varphi}$. Consequently, $R \varphi(R / N)$ has finite length [6, Proposition 4.3]. In particular, there exists a power of $x$ such that $x^{l} \varphi(R / N)=0$. Consider the exact sequence

$$
0 \rightarrow R / N \stackrel{x^{l+1}}{\rightarrow} R / N \rightarrow R / R x^{l+1}+N \rightarrow 0 .
$$

The functor $\operatorname{Ext}(-, R / J)$ gives a long exact sequence

$$
\begin{aligned}
\cdots \rightarrow \operatorname{Ext}_{R}^{k}\left(R / R x^{l+1}+N, R / J\right) & \rightarrow \operatorname{Ext}_{R}^{k}(R / N, R / J) \\
& x^{l+1} \operatorname{Ext}_{R}^{k}(R / N, R / J) \rightarrow 0
\end{aligned}
$$


where the last map is given by left multiplication by $x^{l+1}$. Since by [5, Proposition $6], \operatorname{Ext}_{R}^{k}(R / N, R / J)$ is a right finitely generated $R$-module, left multiplication by $x^{l+1}$ is an isomorphism [15]. But

$$
x^{l+1} \cdot \varphi(r)+\operatorname{Im} d_{k-1}^{*}=\varphi\left(r x^{l+1}\right)+\operatorname{Im} d_{k-1}^{*}=r x^{l}(\varphi(x))+\operatorname{Im} d_{k-1}^{*}=0 .
$$

Since $0 \neq \bar{\varphi} \in \operatorname{Ext}_{R}^{k}(R / N, R / J)$, we get a contradiction. Therefore,

$$
\operatorname{Ext}_{R}^{k}(R / N, R / J)=0 .
$$

We note again that $K-\operatorname{dim}(R / N)=1[9$, Corollary 6]. Therefore, since $J(R / N)$ has the Artin-Rees property and $\bar{x}$ is a regular element of $R / N, R / R x+N$ has finite length as an $R$-module. By [5, Proposition 5], $\operatorname{Ext}_{R}^{k}(R / N, R / R x+N)=0$. Our long exact sequence (1) now becomes

$$
\begin{aligned}
\cdots \rightarrow \operatorname{Ext}_{R}^{k}(R / N, R / N) \rightarrow & \operatorname{Ext}_{R}^{k-1}(R / N, R / R x+N) \\
& \rightarrow \operatorname{Ext}_{R}^{k}(R / N, R / N) \stackrel{x}{\rightarrow} \mathrm{Ext}_{R}^{k}(R / N, R / N) \rightarrow 0 .
\end{aligned}
$$

But $\operatorname{Ext}_{R}^{k}(R / N, R / N)$ is a right finitely generated $R / N$-module by [5, Proposition 6] and $\bar{x}$ is a nonzero element of the Jacobson radical of $R / N$. By Nakayama's Lemma [1, Corollary 15.13], $\operatorname{Ext}_{R}^{k}(R / N, R / N)=0$. Let $R_{[P]} \otimes R / N=(R / N)_{[P]}$. The sequence

$$
0 \rightarrow R / N \rightarrow(R / N)_{[P]} \rightarrow K \rightarrow 0
$$

is exact.

Let $k \in K$ such that $\operatorname{Ann}_{R}(R k)$ is a prime ideal $Q$. Since $K$ is an epimorphic image of $(R / N)_{[P]}, K-\operatorname{dim}(R / Q) \leq 1$. If $K-\operatorname{dim}(R / Q)=1$, then by [9, Theorem 5], $Q \in[P]$. Thus, $R_{[P]} \otimes R k \neq 0$. However, since $R_{[P]}$ is a right flat $R$-module [14, Proposition 2.3.5],

$$
0 \rightarrow(R / N)_{[P]} \rightarrow(R / N)_{[P]} \rightarrow R_{[P]} \otimes K \rightarrow 0
$$

implies $R_{[P]} \otimes K=0$, a contradiction. Consequently $K-\operatorname{dim}(R / Q)=0$. Since $J$ has the Artin-Rees property, $K=\underline{\lim } K_{\alpha}$ where each $K_{\alpha}$ has finite length [6, Proposition 4.3]. Applying the functor $\operatorname{Ext}(R / N,-)$ to (2), we get the long exact sequence

$$
\cdots \rightarrow \operatorname{Ext}_{R}^{k}(R / N, R / N) \rightarrow \operatorname{Ext}_{R}^{k}\left(R / N,(R / N)_{[P]}\right) \rightarrow \operatorname{Ext}_{R}^{k}(R / N, K) \rightarrow 0 .
$$

Since $\operatorname{Ext}_{R}^{k}(R / N, R / N)=0$ and

$$
\operatorname{Ext}_{R}^{k}(R / N, K)=\operatorname{Ext}_{R}^{k}\left(R / N, \underline{\lim } K_{\alpha}\right)=\underline{\lim } \operatorname{Ext}_{R}^{k}\left(R / N, K_{\alpha}\right)=0
$$

[5, Proposition 4 and Proposition 5], we get that $\operatorname{Ext}_{R}^{k}\left(R / N,(R / N)_{[P]}\right)=0$. By [12, Theorem 11.65],

$$
\operatorname{Ext}_{R}^{k}\left(R / N,(R / N)_{[P]}\right)=\operatorname{Ext}_{R_{[P]}}^{k}\left((R / N)_{[P]},(R / N)_{[P]}\right)
$$

But $N_{[P]}$ is the Jacobson radical of $R_{[P]}$. By [3, Lemma 3], $\operatorname{gl} \operatorname{dim}\left(R_{[P]}\right)=$ inj $\operatorname{dim}\left((R / N)_{[P]}\right) \leq k-1$. Therefore, by induction, $K-\operatorname{dim}(R)=K-\operatorname{dim}\left(R_{[P]}\right)+$ $1=\operatorname{gl} \operatorname{dim}\left(R_{[P]}\right)+1 \leq k=\mathrm{gl} \operatorname{dim}(R)$.

One of the most important classes of FBN rings with enough clans are those FBN rings $R$ that satisfy the condition of incomparability of the prime ideals of $R$ with the prime ideals in the center of $R$. In particular, 
COROLLARY 2. If an FBN ring $R$ satisfies incomparability with respect to its center, then $K-\operatorname{dim}(R) \leq \mathrm{gl} \operatorname{dim}(R)$. In particular, this is true for a noetherian ring integral over its center.

Proof. [9, Proposition 9] and Theorem 1.

We should remark that there are examples of noetherian PI rings that do not have enough clans. See for example [9, Counterexamples 1 and 2].

\section{REFERENCES}

1. F. W. Anderson and K. R. Fuller, Rings and categories of modules, Graduate Texts in Math., Springer-Verlag, Berlin and New York, 1974.

2. H. Bass, On the ubiquity of Gorenstein rings, Math. Z. 82 (1963), 8-28.

3. M. Boratynski, A change of rings theorem and the Artin-Rees property, Proc. Amer. Math. Soc. 53 (1975), 307-310.

4. R. Damiano and Z. Papp, On consequences of stability, Comm. Algebra 9 (1981), 747-764.

5. - Stable rings with finite global dimension, Hudson Symposium on Recent Advances in Non-Commutative Ring Theory at Plattsburgh, New York, Springer-Verlag, Berlin and New York.

6. A. V. Jategaonkar, Injective modules and localization in noncommutative noetherian rings, Trans. Amer. Math. Soc. 190 (1974), 109-123.

7. - Relative Krull dimension and prime ideals in right noetherian rings, Comm. Algebra (2) (1974), 429-468.

8. S. Jondrup, Homological dimensions of some P.I. rings, Comm. Algebra 8 (1980), 685-696.

9. B. Mueller, Localization in fully bounded noetherian rings, Pacific J. Math. 67 (1976), 233-245.

10. - Localization of non-commutative noetherian rings at semiprime ideals, Lecture notes, McMaster Univ., 1975.

11. R. Resco, L. Small and J. T. Stafford, Krull and global dimensions of semiprime noetherian $P I$-rings (preprint).

12. J. J. Rotman, An introduction to homological algebra, Academic Press, New York, 1980.

13. J. Shapiro, $R$-sequences in fully bounded noetherian rings Comm. Algebra 7 (1979), 819-831.

14. B. Stenström, Rings of quotients, Springer-Verlag, Berlin and New York, 1975.

15. D. Ž. Djoković, Epimorphisms of modules which must be isomorphisms, Canad. Math. Bull. 16 (1973), 513-515.

Department of Mathematics, George Mason UniVersity, Fairfax, Virginia 22030 